\title{
Botulinum toxin-A for the treatment of neuralgia: a systematic review and meta-analysis
}

This article was published in the following Dove Press journal: Journal of Pain Research

\author{
Fan Meng \\ Ke Peng \\ Jian-Ping Yang \\ Fu-Hai Ji \\ Fan Xia \\ Xiao-Wen Meng
}

Department of Anesthesiology, First Affiliated Hospital of Soochow University, Suzhou, Jiangsu, China
Correspondence: Ke Peng; Jian-Ping Yang Department of Anesthesiology, First Affiliated Hospital of Soochow University, No. 188 Shizi Street, 215006 Suzhou, Jiangsu, China

Tel +86 I59 62I5 5989;

+86 I77 I266 I266

Email pengke0422@I63.com; szyangjp@ suda.edu.cn
Aim: This meta-analysis was performed to evaluate the efficacy and safety of botulinum toxin-A (BTX-A) for the treatment of neuralgia.

Methods: We searched PubMed, EMBASE, and Cochrane databases to identify randomized controlled trials (RCTs) comparing BTX-A treatment with saline for alleviating neuropathic pain. Primary outcome measures were pain scores up to 24 weeks after treatment. Secondary outcomes were hours of sleep, Short Form-36 (SF-36) life quality questionnaire, and adverse events. We used Review Manager 5.3 for the data analyses.

Results: Twelve RCTs were included ( $\mathrm{n}=495)$. Pain scores in the BTX-A group were significantly lower compared to the saline group at 4 weeks (mean difference $[\mathrm{MD}]=-1.64,95 \% \mathrm{CI}$ $[-3.21,-0.07], P=0.04), 12$ weeks $(\mathrm{MD}=-1.49,95 \% \mathrm{CI}[-2.05,-0.93], P<0.00001)$, and 24 weeks ( $\mathrm{MD}=-1.61,95 \% \mathrm{CI}[-2.81,-0.40], P=0.009)$. There were no significant differences in hours of sleep, SF-36 questionnaire, or the incidence of injection pain or hematoma between the two groups. No serious adverse events associated with BTX-A were noted. Fourteen out of 108 patients $(12.9 \%)$ with trigeminal neuralgia experienced mild facial asymmetry after the BTX-A treatment.

Conclusion: Based on the current evidence, BTX-A may be an effective and safe option for the treatment of neuralgia. Due to the limited number of patients included in this meta-analysis, more trials are still needed to confirm these results.

Keywords: botulinum toxin, neuralgia, neuropathic pain, meta-analysis

\section{Introduction}

Clinical examples of neuropathic pain include trigeminal neuralgia, diabetic neuropathic pain, postherpetic neuralgia, and postsurgical neuralgia. Of note, neuropathic pain is often difficult to treat and can lead to anxiety and depression, which seriously compromise patients' quality of life. ${ }^{1}$

Over the years, the pharmacotherapies used for neuropathic pain include pregabalin, gabapentin, antidepressants, anticonvulsants, carbamazepine, lamotrigine, opioids, and so on. However, the evidence for these interventions is often inconclusive. ${ }^{2}$ In addition, side effects associated with the medications such as dizziness, ataxia, nausea, vomiting, somnolence, and rash can be troublesome and debilitating. ${ }^{3}$

Botulinum toxin-A (BTX-A), a potent neurotoxin produced by Clostridium botulinum, blocks acetylcholine release at neuromuscular junctions and causes muscle relaxation. ${ }^{4}$ Animal studies have indicated several possible mechanisms for the analgesic effects of BTX-A: 1) it inhibits the release of pain mediators from both motor and 
sensory neurons, and blocks the release of calcitonin generelated peptide and other neuropeptide $; 5$ 2) it reduces chronic inflammation and acute injury by inhibiting neurotransmitter release, ${ }^{6}$ and 3 ) it deactivates the sodium channel in central nerve system neurons. ${ }^{7}$

Recent studies have shown promising analgesic effects of BTX-A on postherpetic neuralgia, trigeminal neuralgia, and other types of neuralgia. ${ }^{8-19}$ However, sample sizes of the previous studies are relatively small. In this systematic review and meta-analysis, we aim to evaluate the safety and efficacy of BTX-A in the treatment of neuralgia based on the evidence from randomized controlled trials (RCTs).

\section{Methods}

\section{Search strategy}

This study adheres to the guidelines of PRISMA statement and the recommendations of the Cochrane Collaboration (Table S1)..$^{20,21}$

FM and KP independently searched PubMed, EMBASE, and Cochrane databases without language or publication date restrictions. The search strategies are shown in Table S2. Additional articles in the references and reviews were manually searched.

\section{Inclusion criteria}

The studies that fulfilled the following criteria were included in the meta-analysis:

1. Study design: RCT;

2. participants: adult patients with neuralgia;

3. interventions: use of BTX-A compared to saline;

4. outcome measures: pain scores, hours of sleep, the Short Form-36 (SF-36) life quality questionnaire, and adverse events.

\section{Exclusion criteria}

The studies that met the following criteria were excluded:

1. Insufficient data;

2. case reports, reviews, abstracts, editorials, or letters;

3 . in vitro or animal experiments.

\section{Data extraction}

FM and KP independently screened the articles and extracted the following data: first author, publication date, country, sample size, groups and interventions, dose of BTX-A, and main outcomes. Any disagreement over study selection or data extraction was resolved by group discussion.

\section{Primary and secondary outcomes}

The primary outcome was the pain scores in visual analog scale (VAS) or numerical rating scale (NRS) ${ }^{22}$ at 4, 12, and 24 weeks after treatment $(0=$ no pain, $10=$ maximum pain imaginable). The secondary outcomes were changes in hours of sleep and SF-36 at 12 weeks, and adverse events associated with the procedure.

\section{Quality assessment}

FM and KP independently assessed the risk of bias of the included studies using the Cochrane Collaboration tool. ${ }^{21}$ This tool includes seven areas: the generation of random sequence, allocation concealment, participant blind method, result evaluation blind method, data of incomplete results, report of selected results, and other potential sources of bias. For each area, the risk was rated as "high", "low", or "unclear". Any disagreement over quality assessment was resolved by group discussion.

\section{Statistical analysis}

Data analyses were performed using Review Manager 5.3 (The Cochrane Collaboration, Copenhagen, Denmark). For continuous variables, mean differences (MDs) with 95\% CIs were calculated, and for dichotomous variables, ORs with 95\% CIs were calculated. $I^{2}$ was used to evaluate heterogeneity, with $I^{2}>50 \%$ indicating significant heterogeneity. ${ }^{23}$ In view of the clinical heterogeneity, we applied a randomeffects model in this meta-analysis. ${ }^{24}$ A $P$-value of $<0.05$ denoted statistical significance.

\section{Results}

\section{Literature search and study characteristics}

The PRISMA flow diagram is shown in Figure 1. Of the 211 articles initially identified, 56 duplicates were excluded. After reviewing the titles and abstracts, 38 articles remained. Finally, 12 RCTs were included in this meta-analysis. ${ }^{8-19}$ The study characteristics are presented in Table 1. A total of 495 patients (266 in the BTX-A group and 229 in the saline group) were followed up for 8-24 weeks after the treatment.

\section{Risk-of-bias assessment}

The risk-of-bias assessment is summarized in Figure 2. All trials were randomized and double-blind. Two studies did not detail the random sequence generation, ${ }^{11,17}$ and two did not adequately report the allocation concealment. ${ }^{11,15}$ 


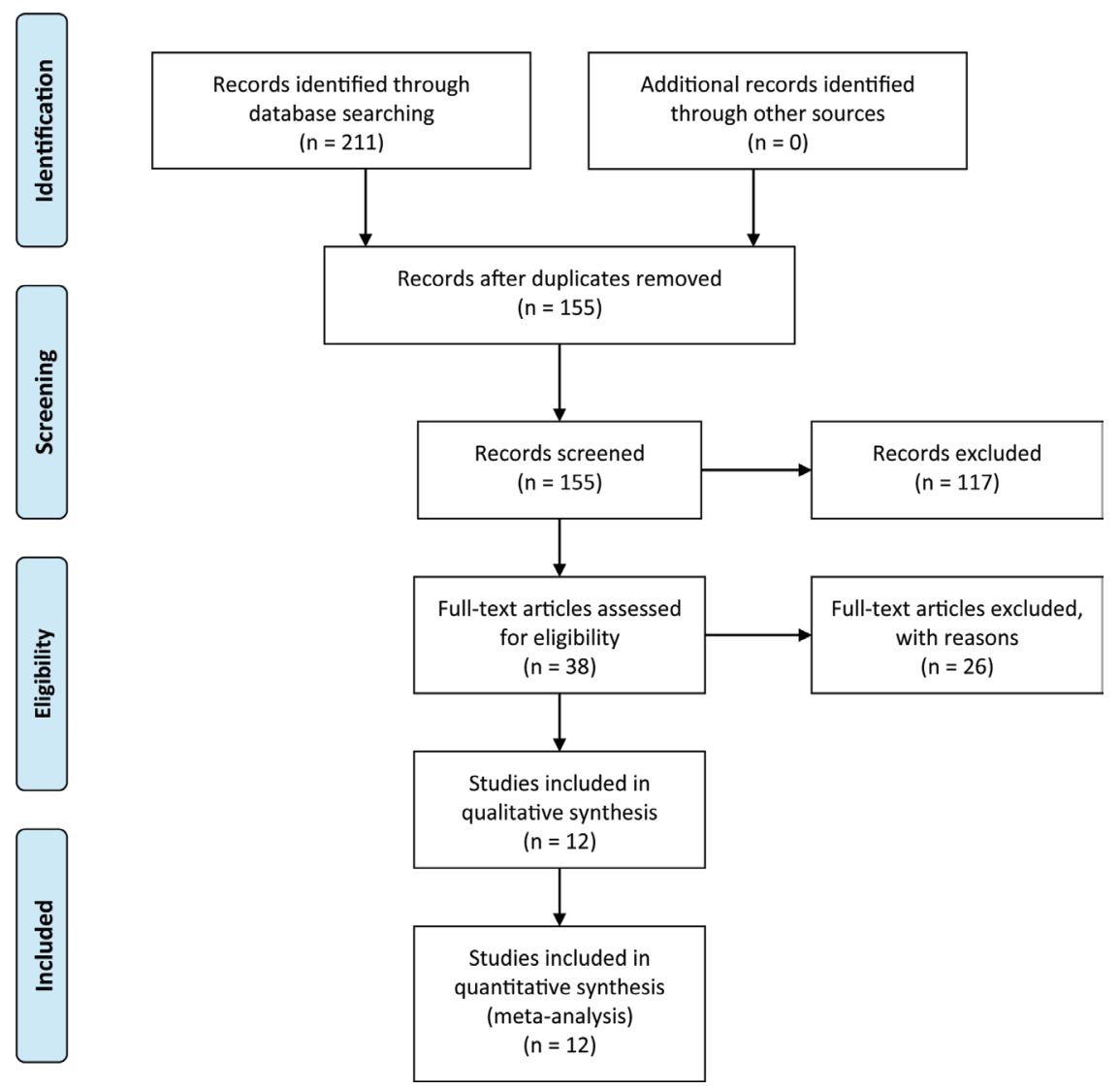

Figure I PRISMA flow diagram.

Note: Copyright @ 2009. PLOS. Adapted from Moher D, Liberati A, Tetziaff J, Altman DG. The PRISMA Group. Preferred Reporting Items for Systematic Reviews and Meta-Analyses: the PRISMA statement. PLoS Med. 2009;6(6):e1000097.33

\section{Pain scores}

As shown in Figure 3, the BTX-A group had significantly lower pain scores than the saline group at 4 weeks (MD $=-1.64,95 \%$ CI $[-3.21,-0.07], P=0.04), 12$ weeks (MD $=-1.49,95 \% \mathrm{CI}[-2.05,-0.93], P<0.00001)$, and 24 weeks $(\mathrm{MD}=-1.61,95 \% \mathrm{CI}[-2.81,-0.40], P=0.009)$.

\section{Secondary outcomes}

As shown in Figure 4, there were no significant differences in changes in hours of sleep (MD $=0.37,95 \%$ CI $[-0.28$, 1.02], $P=0.27$ ) or $\mathrm{SF}-36$ scores $(\mathrm{MD}=52.50,95 \% \mathrm{CI}[-35.88$, 140.87], $P=0.24$ ) at 12 weeks between the two groups.

The incidences of adverse events are presented in Figure 5. No significant difference in injection pain (OR $=0.89,95 \%$ CI $[0.48,1.64], P=0.71)$ or hematoma (OR $=0.93,95 \%$ CI $[0.24,3.67], P=0.92)$ was found between the groups. However, 14 out of 108 (12.9\%) patients with trigeminal neuralgia reported mild facial asymmetry after BTX-A treatment (OR $=7.5,95 \%$ CI [1.64, 34.21], $P=0.009$ ).

\section{Discussion}

In this study, we comprehensively summarized the current evidence and found lower pain scores up to 24 weeks after BTX-A treatment compared with saline in patients with neuropathic pain. Additionally, there were no serious adverse events associated with BTX-A injections.

Recently, there are several published meta-analyses regarding the use of BTX-A in neuralgia. Morra et $\mathrm{al}^{25}$ evaluated BTX-A therapy in trigeminal neuralgia, suggesting that BTX-A may be an effective and safe option. However, only four RCTs were included in their study. Shackleton et al ${ }^{3}$ analyzed six RCTs and showed that BTX-A alleviated trigeminal neuralgia and postherpetic neuralgia. Another meta-analysis by Lakhan et al ${ }^{26}$ indicated that BTX-A treatment improved diabetic neuropathic pain with the results of only two trials. In our study, we included 12 RCTs to determine the effects of BTX-A treatment at three different time points $(4,12$, and 24 weeks). Our results suggested that BTX-A treatment could relieve neuropathic pain both in the short term and in the long run. 


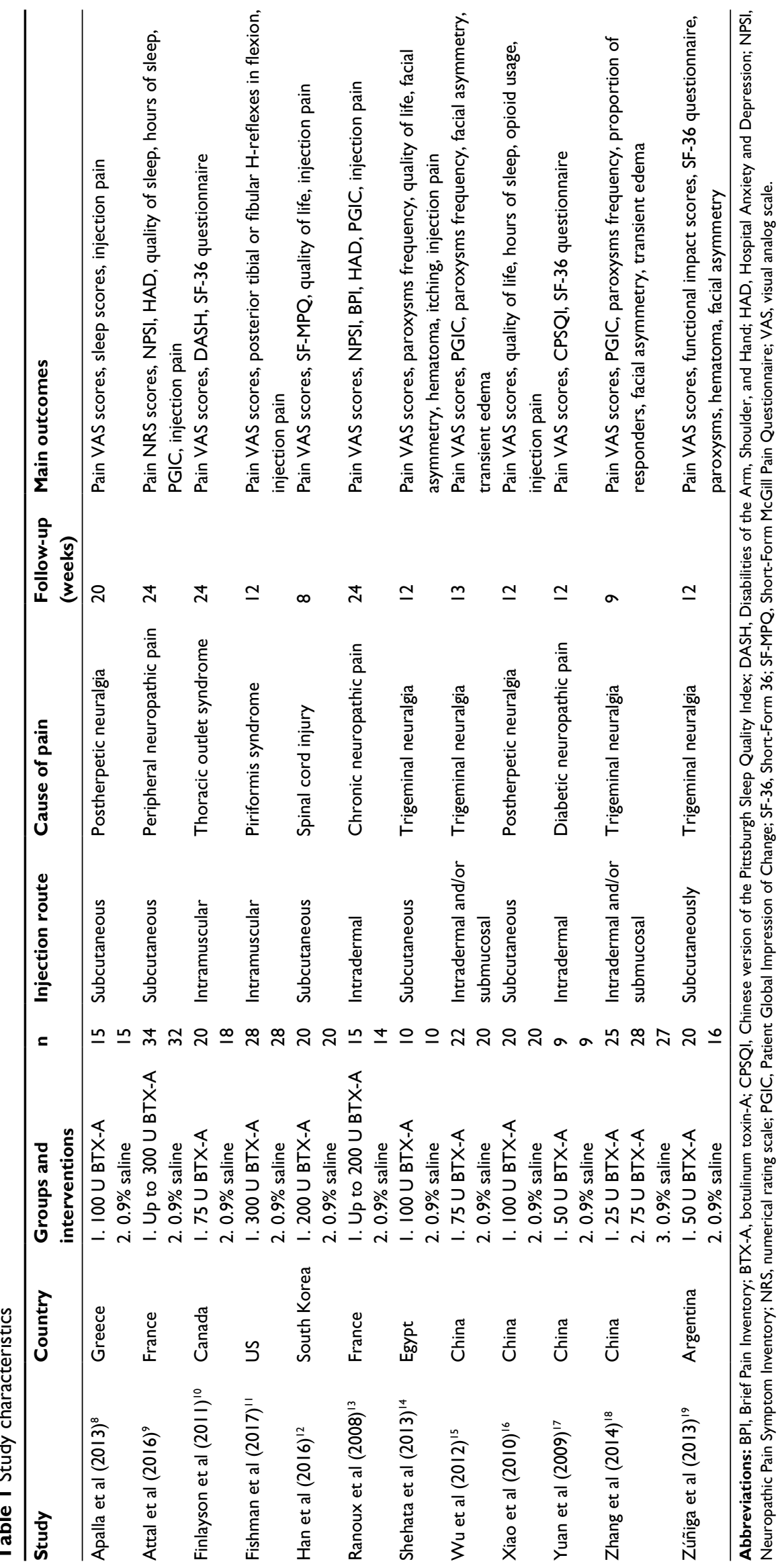



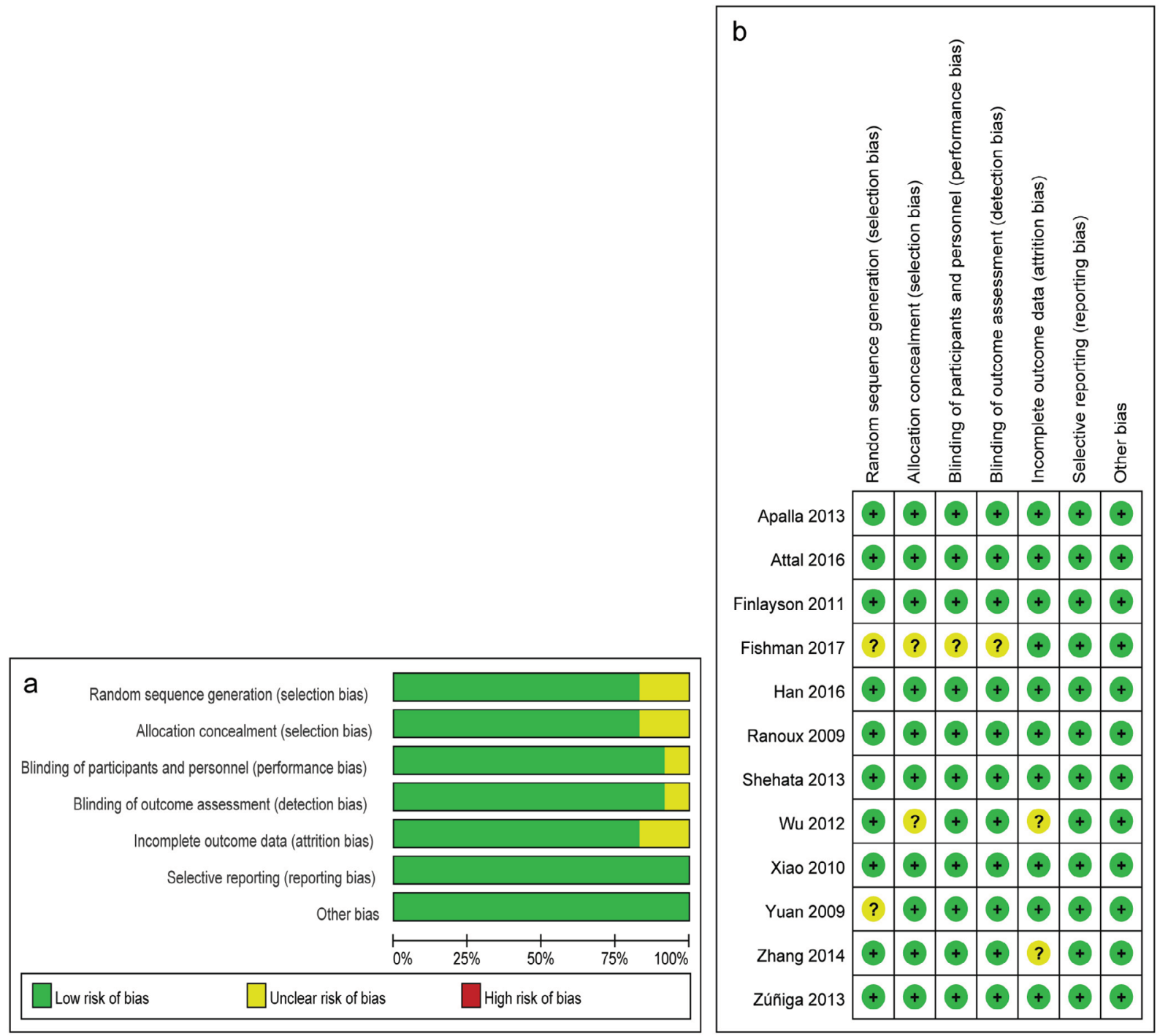

Figure 2 Cochrane risk-of-bias assessment: (A) risk-of-bias graph; and (B) risk-of-bias summary.

This meta-analysis included studies of trigeminal neuralgia, postherpetic neuralgia, diabetic neuropathic pain, and other types of chronic neuropathic pain (peripheral neuropathic pain, thoracic outlet syndrome, piriformis syndrome, and spinal cord injury). Of these, piriformis syndrome is a myofascial condition caused by a nerve entrapment where BTX-A injections reduce the pain by relieving muscle spasms. In cases of other conditions responsible for neuropathic pain including occipital neuralgia, carpal tunnel syndrome, and phantom limb pain, BTX-A was also reported to help reduce the pain. For six patients with occipital neuralgia, BTX-A improved the sharp/shooting type of pain. ${ }^{27}$ One report showed that BTX-A relieved symptoms in five women with carpal tunnel syndrome suggesting the long-lasting antinociceptive effects of BTX-A, ${ }^{28}$ while another study found it did not provide significant relief of carpal tunnel syndrome symptoms. ${ }^{29}$ For amputee patients, BTX-A resulted in improvement of residual limb pain and pain tolerance. ${ }^{30}$ However, no RCT for these types of neuropathic pain could be identified through our literature search.

The injection dose of BTX-A varied among the included studies. Most patients received a dose of 100-200 U, subcutaneously, intradermally, or intramuscularly. For patients with postherpetic neuralgia and trigeminal neuralgia, subcutaneous or intradermal injections of BTX-A were used, which mainly acted by blocking peripheral nerve endings. For other types of chronic neuropathic pain, intramuscular injections were carried out to help relieve muscle spasms. ${ }^{4}$ 


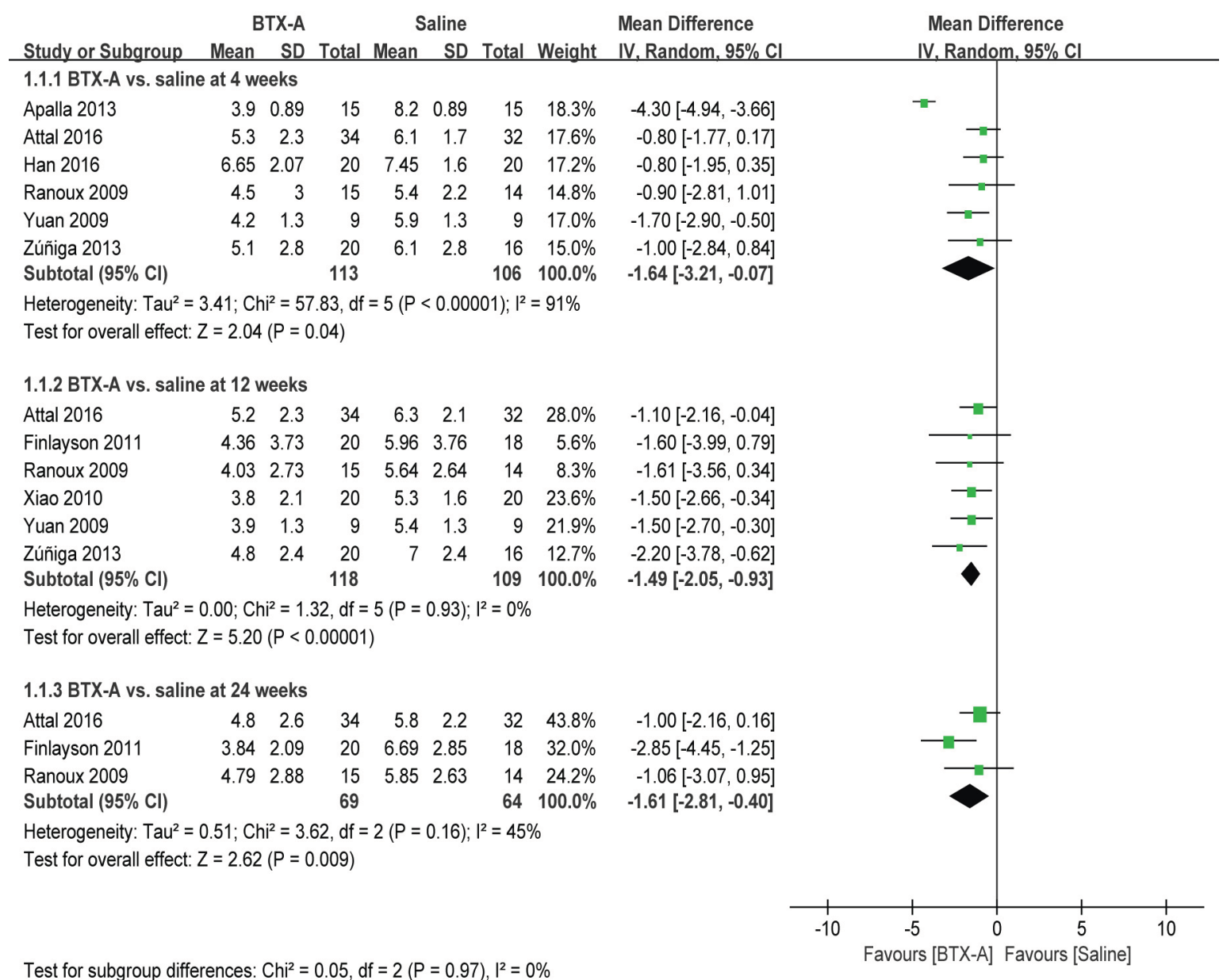

Figure 3 BTX-A vs saline for the treatment of neuralgia: pain scores at 4, 12, and 24 weeks. Abbreviations: BTX-A, botulinum toxin-A; IV, inverse variance.

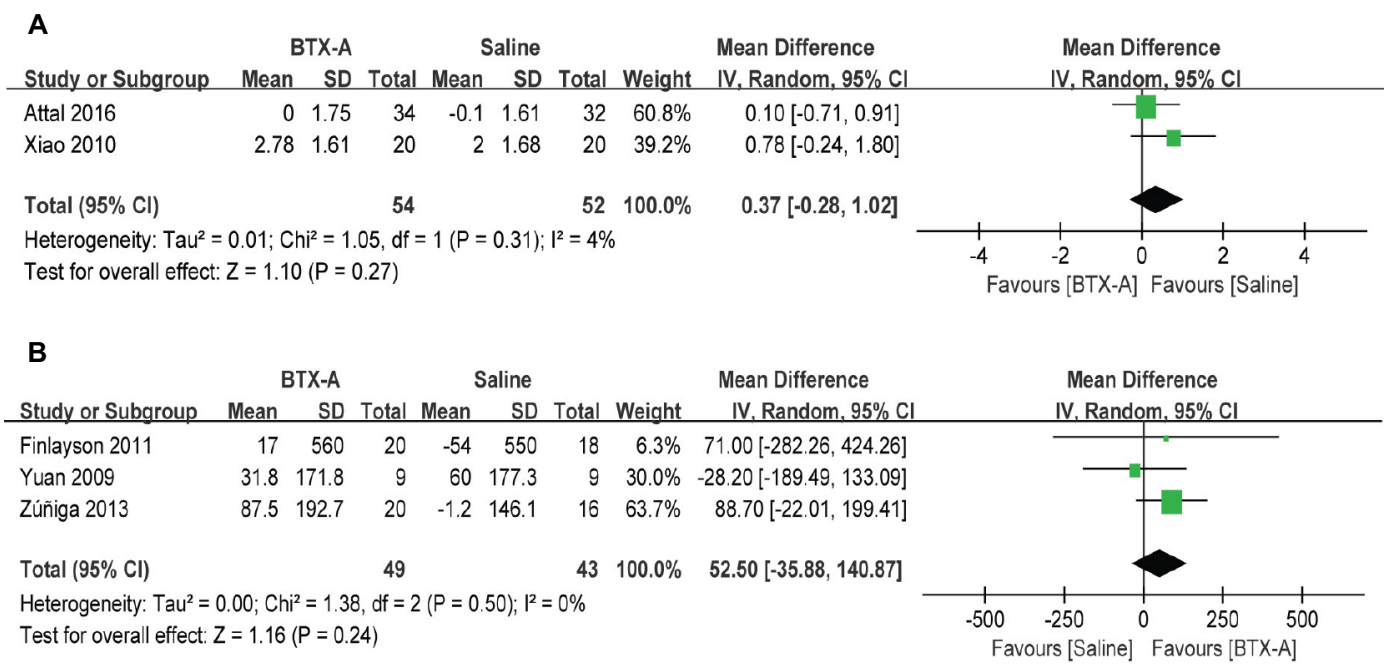

Figure 4 BTX-A vs saline for the treatment of neuralgia: (A) changes in hours of sleep at 3 months; and (B) changes in Short Form-36 questionnaires at 12 weeks. Abbreviations: BTX-A, botulinum toxin-A; IV, inverse variance. 


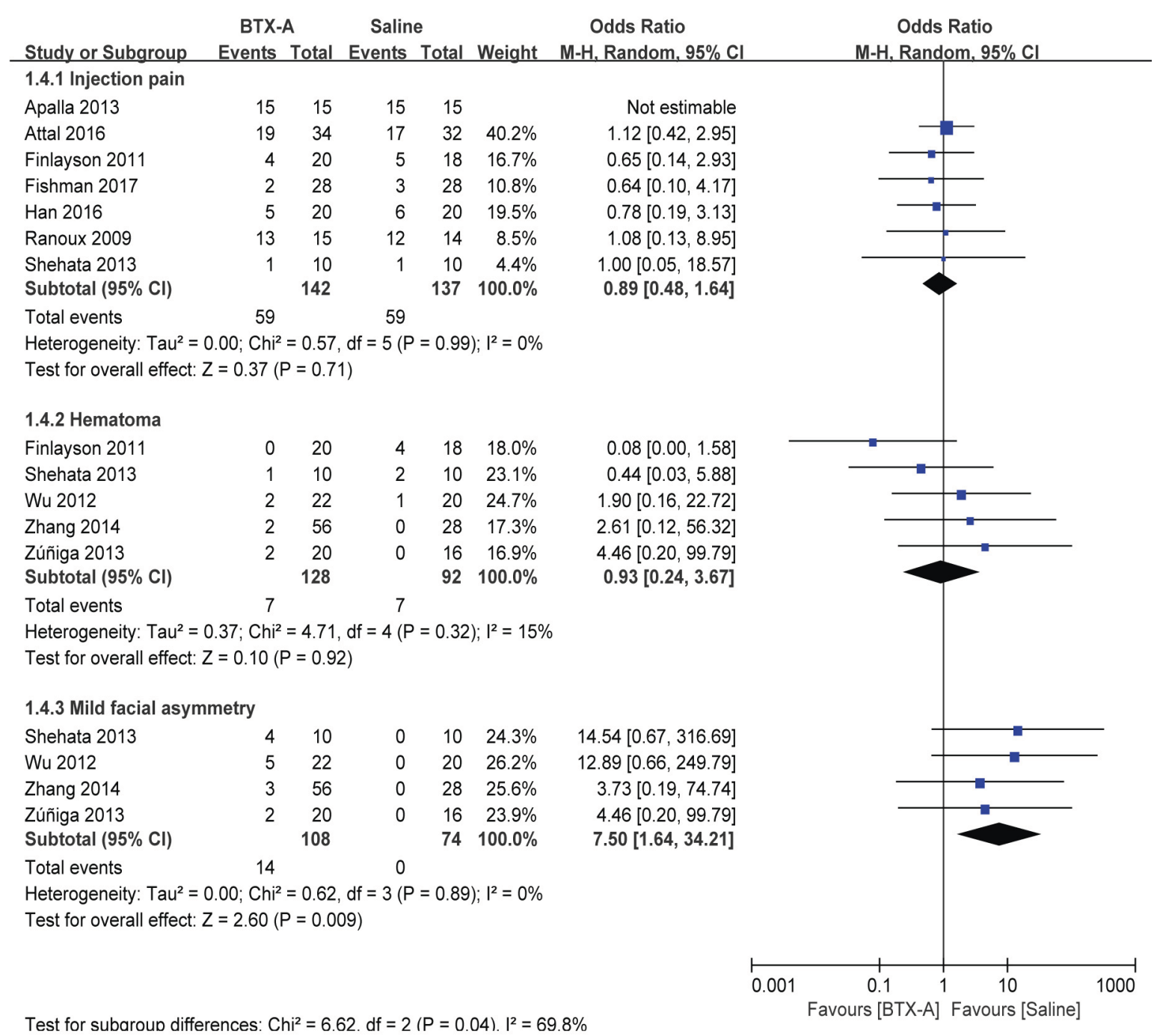

Figure 5 BTX-A vs saline for the treatment of neuralgia: adverse events.

Abbreviations: BTX-A, botulinum toxin-A; M-H, Mantel-Haenszel.

In our study, BTX-A injections failed to improve hours of sleep or quality of life surveyed by SF-36 questionnaire in patients with neuropathic pain. These results did not parallel with the improvement of pain scores after BTX-A treatment. Apart from the limited number of studies included for these two measurements, the complexity of contributory factors in life quality should also be noted for this inconsistency.

Regarding the safety of BTX-A, no significant adverse effects were noted. Overall, 59 out of $142(41.5 \%)$ patients in the BTX-A group and 59 out of 137 (43.1\%) patients in the saline group reported injection pain. Besides, there were seven out of $128(5.5 \%)$ and seven out of $92(7.6 \%)$ patients who exhibited hematoma at injection site. Out of 108 patients with trigeminal neuralgia, 14 (12.9\%) developed mild facial asymmetry after the BTX-A treatment. However, this symp- tom was self-limited without the need for other interventions. Compared to trigeminal neuralgia attacks, patients generally reported this complication tolerable.

\section{Limitations}

There are several limitations of this study. First, the current literature was limited, and the sample size was relatively small. Second, we were not able to explore the potential publication bias through funnel plot due to the insufficient number of studies. Third, some studies suggested that NRS and VAS for pain are not necessarily interchangeable in some clinical situations; ${ }^{31,32}$ thus, potential bias may exist by pooling and analyzing these data. Next, heterogeneity exists for some of the results, so they should be interpreted with caution. Last, our study was underpowered to detect any effect of BTX-A on patients' quality of life. 


\section{Conclusion}

This study suggests that BTX-A may be a good choice for the treatment of neuralgia. More well-designed RCTs with large sample sizes are needed to confirm these findings.

\section{Acknowledgments}

This work was supported, in part, by grants from the National Natural Science Foundation of China (81601659 to KP, 81471835 and 81671880 to FHJ) and the Jiangsu Provincial Medical Youth Talents Program (QNRC2016741 to KP).

\section{Disclosure}

The authors report no conflicts of interest in this work.

\section{References}

1. Baron R, Binder A, Wasner G, Diagnosis Neuropathic Pain: Pathophysiological mechanisms, and treatment. Lancet Neurol. 2010;9(8):807-819.

2. Dosenovic S, Jelicic Kadic A, Miljanovic M, et al. Interventions for Neuropathic Pain: An Overview of Systematic Reviews. Anesth Analg. 2017;125(2):643-652.

3. Shackleton T, Ram S, Black M, Ryder J, Clark GT, Enciso R. The efficacy of botulinum toxin for the treatment of trigeminal and postherpetic neuralgia: a systematic review with meta-analyses. Oral Surg Oral Med Oral Pathol Oral Radiol. 2016;122(1):61-71.

4. Sandrini G, de Icco R, Tassorelli C, Smania N, Tamburin S. Botulinum neurotoxin type $\mathrm{A}$ for the treatment of pain: not just in migraine and trigeminal neuralgia. J Headache Pain. 2017;18(1):38.

5. Durham PL, Cady R. Insights into the mechanism of onabotulinumtoxinA in chronic migraine. Headache. 2011;51(10):1573-1577.

6. Lucioni A, Bales GT, Lotan TL, Mcgehee DS, Cook SP, Rapp DE. Botulinum toxin type A inhibits sensory neuropeptide release in rat bladder models of acute injury and chronic inflammation. BJU Int. 2008;101(3):366-370.

7. Shin MC, Wakita M, Xie DJ, et al. Inhibition of membrane Na+ channels by A type botulinum toxin at femtomolar concentrations in central and peripheral neurons. $J$ Pharmacol Sci. 2012;118(1):33-42.

8. Apalla Z, Sotiriou E, Lallas A, Lazaridou E, Ioannides D. Botulinum toxin $A$ in postherpetic neuralgia: a parallel, randomized, double-blind, single-dose, placebo-controlled trial. Clin J Pain. 2013;29(10):857-864.

9. Attal N, de Andrade DC, Adam F, et al. Safety and efficacy of repeated injections of botulinum toxin A in peripheral neuropathic pain (BOTNEP): a randomised, double-blind, placebo-controlled trial. Lancet Neurol. 2016;15(6):555-565.

10. Finlayson HC, O'Connor RJ, Brasher PM, Travlos A. Botulinum toxin injection for management of thoracic outlet syndrome: a double-blind, randomized, controlled trial. Pain. 2011;152(9):2023-2028.

11. Fishman LM, Wilkins AN, Rosner B. Electrophysiologically identified piriformis syndrome is successfully treated with incobotulinum toxin a and physical therapy. Muscle Nerve. 2017;56(2):258-263.

12. Han ZA, Song DH, Oh HM, Chung ME. Botulinum toxin type A for neuropathic pain in patients with spinal cord injury. Ann Neurol. 2016;79(4):569-578.

13. Ranoux D, Attal N, Morain F, Bouhassira D. Botulinum toxin type A induces direct analgesic effects in chronic neuropathic pain. Ann Neurol. 2008;64(3):274-283.
14. Shehata HS, El-Tamawy MS, Shalaby NM, Ramzy G. Botulinum toxin-type A: could it be an effective treatment option in intractable trigeminal neuralgia? J Headache Pain. 2013;14:92.

15. Wu CJ, Lian YJ, Zheng YK, et al. Botulinum toxin type A for the treatment of trigeminal neuralgia: results from a randomized, double-blind, placebo-controlled trial. Cephalalgia. 2012;32(6):443-450.

16. Xiao L, Mackey S, Hui H, Xong D, Zhang Q, Zhang D. Subcutaneous injection of botulinum toxin a is beneficial in postherpetic neuralgia. Pain Med. 2010;11(12):1827-1833.

17. Yuan RY, Sheu JJ, Yu JM, et al. Botulinum toxin for diabetic neuropathic pain: a randomized double-blind crossover trial. Neurology. 2009;72(17):1473-1478.

18. Zhang H, Lian Y, Ma Y, et al. Two doses of botulinum toxin type A for the treatment of trigeminal neuralgia: observation of therapeutic effect from a randomized, double-blind, placebo-controlled trial. J Headache Pain. 2014;15(1):65.

19. Zúñiga C, Piedimonte F, Díaz S, Micheli F. Acute treatment of trigeminal neuralgia with onabotulinum toxin A. Clin Neuropharmacol. 2013;36(5):146-150.

20. Moher D, Liberati A, Tetzlaff J, Altman DG, PRISMA Group, Group P, Group P. Preferred reporting items for systematic reviews and metaanalyses: the PRISMA statement. Int J Surg. 2010;8(5):336-341.

21. Higgins J, Green S. Cochrane handbook for systematic reviews of interventions. Cochrane Collaboration. 2011; Version 5.1.0. Available from: http://handbook-5-1.cochrane.org/. Accessed March 19, 2018.

22. Heller GZ, Manuguerra M, Chow R. How to analyze the Visual Analogue Scale: Myths, truths and clinical relevance. Scand J Pain. 2016;13:67-75.

23. Higgins JP, Thompson SG, Deeks JJ, Altman DG. Measuring inconsistency in meta-analyses. BMJ. 2003;327(7414):557-560.

24. Peng K, Chen WR, Meng XW, Zhang J, Ji FH, Fh J. Intra-articular dexmedetomidine in knee arthroscopy: A systematic review and metaanalysis. Sci Rep. 2018;8(1):4089.

25. Morra ME, Elgebaly A, Elmaraezy A, et al. Therapeutic efficacy and safety of Botulinum Toxin A Therapy in Trigeminal Neuralgia: a systematic review and meta-analysis of randomized controlled trials. $J$ Headache Pain. 2016;17(1):63.

26. Lakhan SE, Velasco DN, Tepper D. Botulinum Toxin-A for Painful Diabetic Neuropathy: A Meta-Analysis. Pain Med. 2015;16(9): 1773-1780.

27. Taylor M, Silva S, Cottrell C. Botulinum toxin type-A (BOTOX) in the treatment of occipital neuralgia: a pilot study. Headache. 2008;48(10): $1476-1481$.

28. Tsai CP, Liu CY, Lin KP, Wang KC. Efficacy of botulinum toxin type a in the relief of Carpal tunnel syndrome: A preliminary experience. Clin Drug Investig. 2006;26(9):511-515.

29. Breuer B, Sperber K, Wallenstein S, et al. Clinically significant placebo analgesic response in a pilot trial of botulinum $B$ in patients with hand pain and carpal tunnel syndrome. Pain Med. 2006;7(1):16-24.

30. Wu H, Sultana R, Taylor KB, Szabo A. A prospective randomized double-blinded pilot study to examine the effect of botulinum toxin type A injection versus Lidocaine/Depomedrol injection on residual and phantom limb pain: initial report. Clin J Pain. 2012;28(2):108-112.

31. Hartrick CT, Kovan JP, Shapiro S. The numeric rating scale for clinical pain measurement: a ratio measure? Pain Pract. 2003;3(4):310-316.

32. Lund I, Lundeberg T, Sandberg L, Budh CN, Kowalski J, Svensson E. Lack of interchangeability between visual analogue and verbal rating pain scales: a cross sectional description of pain etiology groups. $B M C$ Med Res Methodol. 2005;5:31.

33. Moher D, Liberati A, Tetziaff J, Altman DG. The PRISMA Group. Preferred Reporting Items for Systematic Reviews and Meta-Analyses: the PRISMA statement. PLoS Med. 2009;6(6):e1000097. 
The Journal of Pain Research is an international, peer reviewed, open access, online journal that welcomes laboratory and clinical findings in the fields of pain research and the prevention and management of pain. Original research, reviews, symposium reports, hypothesis formation and commentaries are all considered for publication
The manuscript management system is completely online and includes a very quick and fair peer-review system, which is all easy to use. Visit http://www.dovepress.com/testimonials.php to read real quotes from published authors. 